NUMber of AtTacks and Their Bearing on the QUhstion OF OPERATIVE INTERFERENCE.

In 40 cases the patients had sutfered from three or more attacks, and in 13 instances the attacks had been so frequent -oiten ten or twelve, and sometimes even more numerousthat no count of them had been kept. In 5 cases there had been only one distinct attack, the operation being performed, as pain, tenderness, and a localised thickening persisted for some time after the subsidence of all the acute symptoms. In one of these, the appendix contained a hard fæcal concretion; in 2 it was completely stenosed and dilated beyond; in the remaining 2 it was bent and bound down by adhesions, so that there would undoubtedly have been a recurrence of the attacks in each instance if it had not been removed. The persistence, therefore, for some weeks or months of a tender swelling or thickening in the situation of the appendix, especially if accompanied by rigidity of the overlying muscles and a varying degree nf pain, may be regarded as an indication for operative interference, even wben a patient has sufferei only from a single attack of appendicitis. In the absence of these symptoms, I should not advocate operation after a first attack, for it is a well-recognised fact that a patient may suffer from a single attack and have no recurrence. The probability of the attack recurring is variously estimated at from 23 to 44 per cent. of all cases. When, however, a patient has suffered from a second attack, operation should be advised even in the absence of any local symptoms during the quiescent periods, for after a second attack more are almost certain to follow.

\section{DURATION OF SYMPTOMg}

The length of time during which the patients had suffered from the attacks varied from several months to six years.

Srx.

Of the 50 patients, 35 were males and 15 were females, it being gentraliy found that the former sex are the most liable to this affection.

AGE.

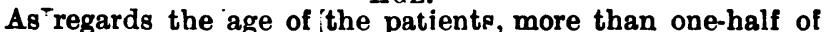
the cases-namely, 29-were met with in persons between 20 and 30 years old, 12, occurred in persons between 10 and 20 7 between 30 and 40 , and 2 between 40 and 50; so that after 30 years of age the tendency to this affection diminishes as life advances. Its more frequent occurrence in young subjects is probably due to the fact-as Kelynack ${ }^{2}$ has pointed ontthat more lymphoid tissue is present in the wall of the appendix in the young than at a more advanced age, and the tendency for this structure to become the seat of inflammatory processes in early life is well recognised.

NOTE AND REFERENCE

1 In ${ }_{4}$ cases operated upon since the completion of the above series, a local abscess was found in 3 instances. This gives a total of 64 cases, in 18 of which suppuration had taken place-that is, in 28 per cent. ${ }^{2}$ The Pathology of the Vermiform Appendix, p. 56 .

\section{QUANTITATIVE COLOUR-TESTS.*}

By KARL GROSSMANN, M.D., F.R.C S.E. Ophthalmic Surgeon to the Stanley Hospital, Liverpool.

More than ten years ago I pointed out the shortcomings of the method of testing the colour sense by means of Holmgren' wools. At the Tentin International Meaical Congress at Berlin in August, 1890 , I described the model of an apparatus constructed for the purpose of obviating those defects. The apparatus itself was constructed soon after, and proved to be of service. A long spell of illness and other causes subsequently prevented me from pursuing the subject, but on resuming it find the matter stands practically to-day where it stood ten years ago.

The insufficiency of Holmgren's test in a number of cases had already been observed by others before 1890 . To mention a minor objection to the test: as used at present everywhere it is only a daylight test. With the variability of the quality of the light on the one hand, and the restriction of its applicability, on the other, owing to the frequency of dark days in this country, a great inconvenience exista. True. a

* Read in the Section of Uphthalmology at the Annual Meeting of the British Medical Association at Ipswich, remedy could be found by selecting a set of colours adapted for a particular artificial light. But the very fact that this has not been done shows how very deep the faith in the infallibility of the Holmgren test is rooted.

Chentrat Colour Scotoma.

There is, however, a very serious objection to the Holmgren est which cannut be overcome by any makeshift or adaptation, but claims our full attention, and that is the occurrence of a central colour scotoma. This is all the more important and dangerous as it need not be a congenital defect, but may be acquired and even transient-for example, in nicotine poizoning. Here past statistics leave us comple tely in the dark and are totally valueless. Sometimes it occurs that the faulty mode of examination (Holmgren) declares $8 \mathrm{uch}$ an individual colour gound, and that practical life then puts him to a more cevere test, showing him to be colour blind for his antual requirements. I will not take any of the older observations of others or myself, but will refer to a case published recently by $\mathrm{Mr}$. Bickerion ${ }^{1}$ : The second officer of a 4,000-ton steamer, when on the bridge, could not distinguish between a green and a white light, and nearly brought about a collision with a sailing vessel, a catastrophe being averted only by the accidental arrival of the captain on deck. Mr. Bickerton relates the case without any comment as to its nature. Professor Lodge, to whom he sent the patient for examination, found only "a slight defect of red sensation" and no explanation is given. Without knowing anything further about the case, it would be surprising indeed to me if this was not a case of central colour scotoma which would have been readily detected by a proper test. The letter by Dr. Hubert Seager, quoted in that article, says:

I do not remember to have yet seen any discussion on the peculiar form of colour blindness to which I myself (and, no doubt, other people as well) am subject-inability to distinguish at night between a red and a green light at a distance exceeding half a mile, though I can pass any test for colour or long sight in daytime, and can distinguish coloured lamps perfectly at night within half a mile's distance. I tested this condition of my eyesight again last year when I was at sea for a week or two, and found it unaltered ...... Naturally such a state of colour blindness might lead to very serious accidents at night, and the Board of Trade tests should be apted to such defects.

Clearly here we have a case of a central colour scotoma in both eyes. A coloured signal sufficiently near is both strong enough and large enough to give a sufficiently large image on the retins, in fact, to exceed the area of the scotoma; if the distance ipcreases both the intensity and the size of the light decrease, and the image falls within the limits of the scotoma, with the result of not being recognised correctly. On the other hand, the Holmgren wool skeins will be easily recognised in such a case. They can be manipulated, and, even if the \&cotoma be fairly large, can be brought near enough to the eye to cover a large area of the vision field. And that is exactly what we do see in some cases of "doubtful" colour blindness.

In order to detect cases of colour blindness of the kind just referred to, the following points have to be taken into consideration. The test object must be :

(a) Variable in colour, that is, a variety of colours must be at hand so as to avoid the learning by heart of three, or four, or five colours only. (b) Variable in size, that is, from a large size down to a very small point. (c) Variable in intensity, that is, it must be possible to make it bright, and through all transitions dark to almost extinction.

This can best be effected by using actual signal lights suitably arranged. An equality of the source of light can easily be obtained as well, a point of great vaive for the comparison of the results. Moreover the examinee is thereby put under precisely the same conditions as be meets in his occupation as geaman or railway employee. If the conditions under $(b)$ and (c) are sufficiently variable, there is no need to vary the distance within which the tests bave to be recognised.

One other point should be observed. Any method in which the examinee has to give an answer to such a question as, "What colour is this?" ought to be avoided. In this respect the principle of matching one colour with a second one, to be selected out of a batch of othere, which underlies the HolmgrenSeebeck test, is paramount.

Uniformity of the source of light being necessary, the examination is carried out in a darkened room, artificial light of a given kind being employed. 
DeSCRIPTION OF TEE INSTRUMENT.

The following is a description of the instrument $I$ have used. A few modifications which will materially reduce the cost of production, and will also be found to be improvements, will be mentioned at the end.

In order to reproduce in the apparatus the actual conditions of practical ife, I took as the source of light an ordinary signalling hand lamp, such as used on some railways (Fig. $x, a)^{*}$ In front of the bull's eye are placed

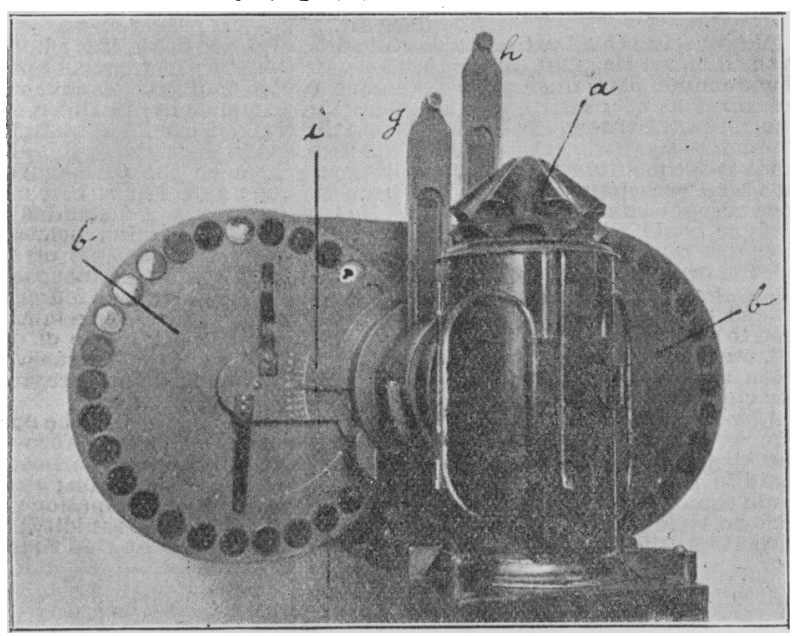

Fig. x.

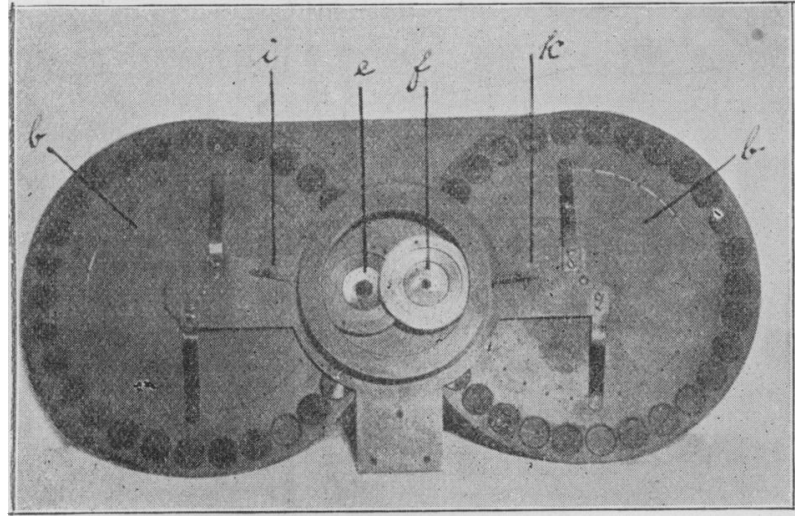

Fig. 2.

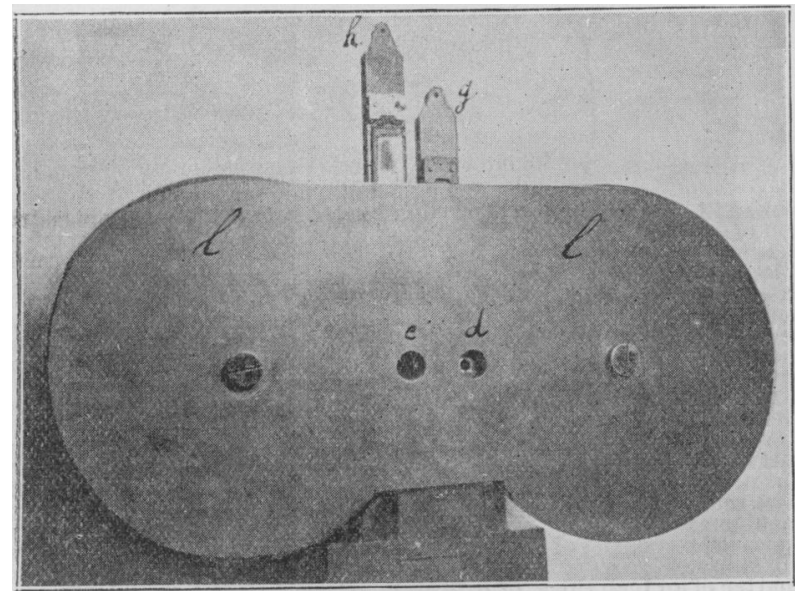

Fig. 3.

two metal discs (Fig. I, $b$ ), capable of being moved round their centres, photographs atren tor bertures flled with glassea of various colours. The colours in each of the discs correspond, though arranged in different order. On the other side of the discs is a metal plate (Fig. $3, l$ ). which overlags them, and is perforated by two holes (Fig. $3, c, d$ ), one boside the other. Each of these holes is so placed, that by rotating the disc on its corresponding side exch of the colour

brought between it a ad the bull's eye of the lantern. Between the bull's eye and each opening in the plate there is an iris diaphragm (Eig. 2, $e, f$ ), with indicators (Fig. 2, $i, k ;$ Fig. $x, i)$ showing the diameter of the apertures in millimetres. [Fig. 2 represents the apparatus

Between the diaphragms and the lamp two slips of glass, suitably mounted, are made to slide up and down. (8ig. $x, g, h$; Fig. $3, g, h$ ) These slips aro tinted neutral groy, tho intels being graduated from complete

They were first made of neutral-tinted glass, ground wedge-shaped, but the great the great cost of such wedges los from ordinary dry plate negatives gres these slips are messured photometrically, and indexed accordingly.

Bg this means the opening for the light falling through a coloured glass can be made to vary in size from $10 \mathrm{~mm}$. diameter to vin point, and in intensity from light to dark. An almost unlimited variety of lights, both coloured and "white," can thus be obtained. Both discs can be moved independently, as can also the diaphragms and glass slips.

For convenience sake I use the mirror method for testing, a method which I have employed for over twenty years in my consulting room for Satlen's letters. I place the instrument on a table, with the mirror in front at 15 feet distant. I am seated to the right of the apparatus, the examinee to the left. I select one of the colours of the right disc, the iris, diaphragm, and tint slip having been suitably adjusted. The examinee is then instructed to turn the left disc 80 as to find a match for the signal light of the right disc. The coloured glasses are partly chosen from various pairs and sets of confars ("twin colours" and "triplet colours" of my previous tests). There are at present thirty in each disc, but half that number will probably be found sufficient.

The graduated neutral tint glass slips will be replaced by disc fix and therefore cheaper, than the sliding glass slips.

The lamp used hithertu has been the colza oil lamp shown in Fig. $1, a$. Although the flame was kept fairly steady, variations in the intensity of the light could not always be avoided, and in future the source of light will be a standard sperm candle, the unit adopted in all countries for measuring the intensity or quality of any light.

Advantages OF the INSTRUMENT.

As already mentioned, I insist upon the matching of colours, and do not ask any question whatever as to their colours, This is a point of paramount importance, which I cannot too strongly insist upon in any colour examination. (For this reason the apparatus just described by Professor Eversbuch in von Graefe's Arch, 1., i, June, 1900, is inadequate.)

If anyone should object to having the two coloured signal lights side by side simultaneously, the one to be compared with the other, since in practical life, at sea and on a railway, with the other, since in practical and interpret a single signal, the apparatus can be used in a corresponaing manner. Either of the lights (Fig. 3,c, $d$ ) can be blocked out by a cover. One cover is removed first, a coloured light shown, and the cover cover is removed first, a coloured light shown, and the cover let down again. The examinee is then asked to match this signal lifted.

By narrowing the diaphragm we are able to detect the smallest central scotoma, the possibility of which has hitherto been completely neglected in all practical tests. They have not been looked for even in examinations with spectroscopic not been loot not that there need have been auy difficulty in instruments; not that there need have been auy diffculty in detecting them, but because their importance, and even their existence, has not been recognised. The selection of twincoloured signals is a saving of tim practical ntility of the apparatus.

The apparatus admits of the adoption of a standard for the quantitative measurement of coluur perception. I have hitherto used as the standard the lamp=i candle power, mm. diaphragm, 50 per cent. of the light obscured, and a distance of 15 feet from the mirror $=$ (10 yards actual distance). I am, however, measuring this again, using the actual I am, howerm candle in place of the colza lamp, and shall 
publish the results as soon as possible. (The coloured glasses must, of course, be of a certain standard quality.)

We may by this means be enabled to form a standard as simple and as useful as Snellen's standard for form vision. With the average normal central colour vision (C. V.) reduced to $\frac{1}{2}$ for red and green, rejection of the examinee is advisable; with $\mathrm{C}$. V. reduced to $\frac{1}{4}$ it becomes an absolute necessity.

To secure accuracy each eye should be examined separately, and, though for practical purposes it would be quite sufficient if one eye onlp satisfied the colour test, still it would be of great importance to know that such and such an individual was suffering from a defect of colour perception in one eye.

REFERENCE.

1 BRITISH MEdicAL JOERNAL, March r7th, 1900.

\section{REMARKS ON CHRONIC ENLARGEMENT OF THE PANCREAS IN ASSOCIATION WITH OR PRODUCING [ATTACKS SIMULATING BILIARY COLIC.*}

BY GILBERT BARLING, M.B., F.R.C.S.,

Co-Professor of Surgery in the University of Birmingham; Surgeon to the General Hospital.

DiSfaSkS of the pancreas are not yet as well understood, or as thoroughly worked out, as diseases of the other abdominal viscers. Two reasons appear to me to explain this in the main : first, the pancreas is not easily examined in the living subject, owing to its depth and the protection given it by the liver and stomach ; and secondly, the examination of the organ at necropsies is often most perfunctory. Evidence of the truth of the latter statement seems to me to be afforded by the condition of acute infective pancreatitis. This disease has only of recent years received recognition, but it is very unlikely to have originated as a new disease in the last few years, and yet if the organ had been thoroughly examined in past times, the striking picture presented by it could hardly have been overlooked.

The purpose of this communication is to present 4 cases of enlargement of the pancreas which I have found accidentally when operating on the gall bladder or ducts. Two of these were distinct errors in diagnosis, for gall stones were not found, although it was believed that they were responsible for the symptoms.

History. -The first case occurred in 1897 in the practice of Dr. N. H. Turner, with whom I saw the patient, a gentleman aged 44, in what appeared to be a characteristic attack of gall stone colic. There was pain in and to the right of the epigastric region, going through to the back, vomiting and distinct jaundice; tenderness also existed over the lower similar attacks at intervals of weeks or months, some of them associated with shivering.

Operation. -I operated for gall stones in April, 1897 , but none existed, Operation.-1 operated for gall stones in April, 1897 , but none existed,
and the only condition found which might explain the attacks was a considerable mass in the head of the pancreas, firm but not very hard, somewhat lobulated on the surface, and occupying such a position that it might easily compress the common bile duct. A guarded prognosis was given, the rriends being told by me that whilst such formations did at growth.

After-History.-A good recovery followed the operation, and for some months the patient considered himself relieved Subsequently the some returned, and the patient at this date, three years and a half after his operation, still suffers from them; ; but he is otherwise well, and evidently his pancreatic enlargement was not due to malignant growth. Soon after the operation, although I knew exactly where to palpate for the tumour, most difficult to relax.

$$
\text { CasE II. }
$$

History. - The second case occurred in a stout woman, aged 53, who was admitted to the General Hospital April 4th, 1899, complaining of attacks of biliary colic The first seizure was in November, 1898, the symptoms being severe pain in the epigastrium, radiating widely over the abdomen, and associated with vomiting. Several minor attacks followed, and a severe one at Christmas, after which four gall stones were discovered in the
motions. On March 25 th a severe attack occurred, and three small stones were passed.

Operalion.- The operation was performed on April pth, r8g9, the gall bladder being only slightly distended with muco-purulent material, and three small gall stones lay near the orifice of the cystic duct. A mass the size of a good orange was felt in the position of the head of the pancreas-
it was firm but not hard, rather lobulated, and fairly movable. Only a slightly bile-stained discharge took place from the gall bladder for more than a month, and then bile escaped copiously.

* Read at a meeting of the Birmingham and Midland Counties Branch of the British Medical Association.
After History. - The drainage tube was retained for a couple of months to ensure complete recovery from the cholecystitis which existed at the of the operation, and it was six months later before the wound (he time of the oxerallent health. Here again I could not with certainty palpate the pancreatic Wargement, buit the thickness of the abdominal walls was considerable. from an inflammatory enlargement of the pancreas and not from growth.

\section{CASE III}

History. -The third patient, a man aged 29, of spare habit, was admitted to the General Hospital, July 29th, 1899 , with a history of frequent attacks of abdominal pain during the preceding twelve months. The pain was described as of a durp pullig characthe ciph line, midway between the umbilcus and the xiphoid cartilage, and going through to the back. Occaslonally the pala was to the right or left of the point above mentioned ; rarely vomitirg accompanied the pain, and only on three occasions had jaundice been noticed, gall stones had never been recognised in the evacuations. No tumour such as a distended gal bladder could with certainty be recognised, but the region below the cussion over the edge of the right erector spinæ in the ilio-costal space. Operation. - At the operation on July 3 st the gall bladder was found disonded, and over 800 stones were removed varying in size from a mustard tended, and over 800 stones were removed varying in size from a mustar, seed to an orange pip. A frm, lobulated mass, nearly the size of one's colon and crossing the front of the vertebral column; this was regarded as a chronically in ilamed pancreas.

After-History. I I did not see the patient for some weeks after the operation, as I was ieaving for my holiday, but he made a very good recovery, and was discharged healed on August 29 th. He came to the hospital again in December 889 , complaining of pain in the epigastrium; a mass could then be felt in the region of the pancreas, and examination from time to time showed that this was enlarging with some rapidity; the patient was therefore readmitted on January 22nd, s9oo. At this time he

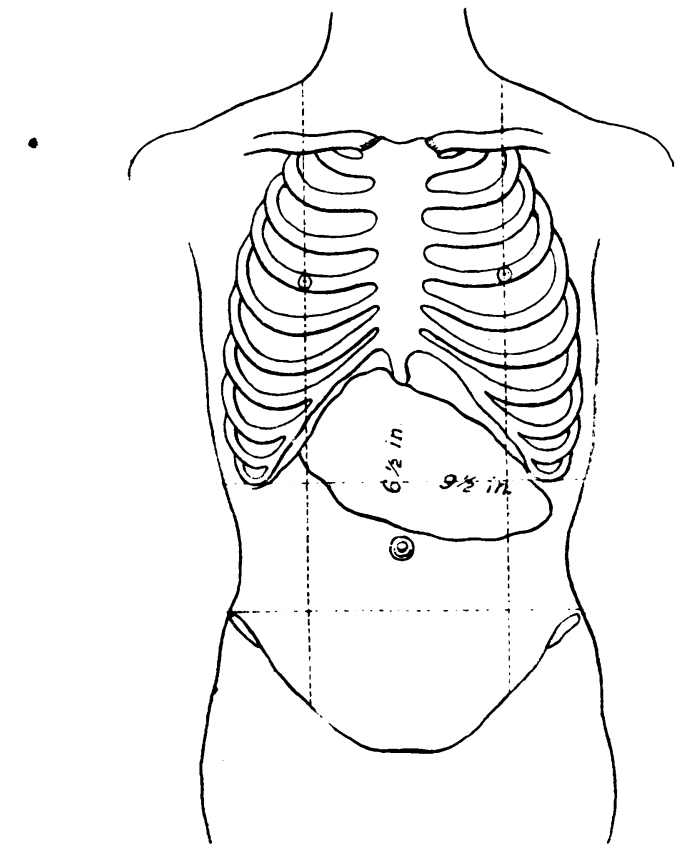

looked ill, was losing weight, complained of pain deep in the epigastrium and of constant nausea and inability to take food. The urine contained a haze of albumen, but was free from sugar; the stools were normal in colour, and did not appear to contain fat. A tumour existed which umbilicus, its long axis was transverse, and it extended somewhat beyond the semilunar line on both sides, being a little more extensive on the left than on the right side ; a thrill could be obtained over it. Examination of the chest posterioriy failed to give any physical signs of the nation of the chest posteriorly falled to give any physical signs of the
tumour. The diagram shows an outline of the cyst, which measured $9 \frac{2}{2}$ inches transversely and 6 i inches vertically.

Second Operation.-The abdomen was opened over the most prominent part of the cyst to the left of the nidddle line; the great omentum, which part of the cyst to the left of the nidadle line; the great omentum, which
was not adherent. was pushed away, and 8o ozs. of dark, greenish fluid was not adherent, was pushed away, and 80 ozs. of dark, greenish fluid
were removed with the aspirator ; the cyst was then opened, and a further considerable quantity of fluid escaped into sponges. The cyst wall appeared to consist of two layers, an outer tough flbrous coat about the thickness of the external oblique aponeurosis, and an inner layer about a quarter of an inch thick, of pale pink, rather friable material. The opening in the cyst was stitched to the peritoneum, and two drains were placed in the cavity. The fluid was, unfortunately, not examined until the next day, so that changes may have occurred in it; the reaction was
then alkaline, and it had neither diastatic nor peptonising power. Atter-History.-In the first twents-four hours there was a profuse dis 